International Journal of Biomedicine | June 2021 - Volume 11, Issue Suppl_1: Abstracts from the Third Russian International Conference "Cryo-electron microscopy 2021: achievements and prospects"

POSTER ABSTRACT PRESENTATIONS

SESSION TITLE: STRUCTURE AND FUNCTIONS OF THE TRANSCRIPTION AND TRANSLATION APPARATUS OF THE CELL

DOI: 10.21103/JJBM.11.Suppl_1.P23

\title{
Abstract P-23: Histone N-terminal Tails Reduce Early Nucleosomal Pausing during Transcription
}

\author{
Nadezhda S. Gerasimova $^{1}$, Fu-Kai Hsieh ${ }^{2}$, Vasily M. Studitsky ${ }^{1,2}$ \\ ${ }^{I}$ Biology Faculty, Lomonosov Moscow State University, Moscow, Russia \\ ${ }^{2}$ Fox Chase Cancer Center, Philadelphia, PA, USA
}

Background: Nucleosomes are the barriers to transcript elongation by RNA polymerase 2 ( $\mathrm{Pol} 2$ ) in vitro and in vivo. Formation and overcoming the barrier are important for transcription regulation. $\mathrm{N}$-terminal tails of core histones do not affect the inner structure of nucleosomal core. However, strongly positively charged tails can interact with the DNA, thereby impeding polymerase progression through the template. Removal of histone tails was shown to facilitate transcription through a nucleosome by both yeast and human Pol 2, and the effect was most noticeable at lower ionic strength $(40 \mathrm{mM} \mathrm{KCl})$. In vivo experiments established a new mechanism of overcoming of +1 nucleosomal barrier by removal of histone tails by specific regulative proteinase. As +1 nucleosomal barrier is formed mostly by the promoter-proximal part of the nucleosomal DNA, here we address the effects of histone tails on elongation through this part of the nucleosome.

Methods: We have studied the effect of histone tails on transcription by yeast Pol 2 and model enzyme E. coli RNA polymerase utilizing very similar mechanisms of elongation through chromatin. 603 nucleosomes were transcribed in vitro using purified proteins and components. To focus on the proximal part of the nucleosome, transcript elongation was conducted for a limited time and at low ionic strength.

Results: For the phosphorylated form of yeast Pol 2 and E. coli RNAP, histone tail removal significantly reduces the strong nucleosome-specific pausing that the yeast polymerase encounters $\sim 15$ bp within the 603 nucleosome and further downstream, leading to both increased traversal of the pause and the accumulation of complexes paused at more distal locations. However, tail 
removal did not lead to a significant increase in full traversal of either nucleosomal template. The effect of histone tails removal was cognate for both enzymes but differs in detailed effect on the barrier.

Conclusion: Histone tails provide a significant part of the nucleosomal barrier to transcript elongation by Pol 2-type mechanism. The effect is very pronounced in the promoter-proximal part of the nucleosomal DNA, suggesting that histone tails could play a role during the regulation of the +1 nucleosomal barrier. The role of Pol 2 CTD phosphorylation and formation of the intranucleosomal loops in the regulation of +1 nucleosomal barrier will also be addressed.

Key Words: RNA polymerase $\bullet$ transcription $\bullet$ nucleosome $\bullet$ histone tails

This work was supported by the Russian Scientific Foundation (Grant No. 1944-02013)

*Corresponding author: Nadezhda S. Gerasimova. E-mail: gerasimova@mail.bio.msu.ru

International Journal of Biomedicine. 2021;11 Suppl 1: S21.

doi: 10.21103/IJBM.11.Suppl_1.P23

(C)2021 International Medical Research and Development Corporation 